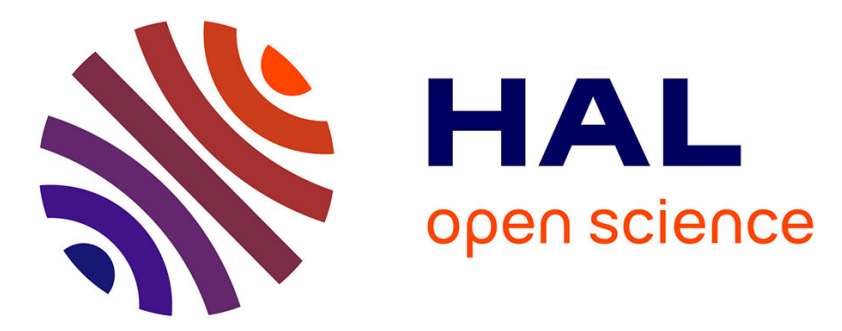

\title{
Directional and Selective Mid-Infrared Thermal Emitters for Sensing Applications
}

Cédric Blanchard, Q. Lévesque, D. Costantini, Cécile Jamois, Jean-Louis Leclercq, A. Coutrot, F. Marquier, Laurent Milord, Christian Grillet, H. Benisty, et al.

\section{To cite this version:}

Cédric Blanchard, Q. Lévesque, D. Costantini, Cécile Jamois, Jean-Louis Leclercq, et al.. Directional and Selective Mid-Infrared Thermal Emitters for Sensing Applications. Advanced photonics 2015, Optical Sensors, Jun 2015, Boston, United States. pp.SeW2B.2, 10.1364/SENSORS.2015.SeW2B.2 . hal-01489577

\section{HAL Id: hal-01489577 https://hal.science/hal-01489577}

Submitted on 30 Jun 2021

HAL is a multi-disciplinary open access archive for the deposit and dissemination of scientific research documents, whether they are published or not. The documents may come from teaching and research institutions in France or abroad, or from public or private research centers.
L'archive ouverte pluridisciplinaire HAL, est destinée au dépôt et à la diffusion de documents scientifiques de niveau recherche, publiés ou non, émanant des établissements d'enseignement et de recherche français ou étrangers, des laboratoires publics ou privés. 


\title{
Directional and Selective Mid-Infrared Thermal Emitters for Sensing Applications
}

\author{
C. Blanchard $\dagger \neq$, Q. Lévesque $\ddagger$, D. Costantini $\ddagger$, C. Jamois $\dagger$, J.-L. Leclercq $\dagger$, A.-L. \\ Coutrot $\ddagger$, F. Marquier $\ddagger$, L. Milord $\dagger$, C. Grillet $\dagger$, H. Benisty $\ddagger$, P. Viktorovitch $\dagger$, X. \\ Letartre $\dagger$, J.-J. Greffet $\ddagger$ \\ $\dagger$ Institut des Nanotechnologies de Lyon. École Centrale de Lyon. 36 av. Guy de Collongue, 69134 Ecully, France. \\ $\ddagger$ Laboratoire Charles Fabry, Institut d'Optique Graduate School. 2 av. Augustin Fresnel, 91127 Palaiseau, France.
}

cedric.blanchard@institutoptique.fr

\begin{abstract}
We propose energy efficient gas sensors that are based on the manipulation of the spectral and spatial emission of incandescent sources. To do so either photonic or plasmonic resonances in texturized nanostructures are employed.
\end{abstract}

Keywords: Sensing, thermal radiation, metastructure.

\section{Introduction}

We are currently witnessing the emergence of a new frontier in scientific research through the prominence that is given to the development of new concepts and technology enabling a drastic reduction in energy consumption. Some of these new concepts aim at rising a given functionality by employing a device for which the dispersion characteristics are precisely controllable. For the sake of illustration we may mention the thermophotovoltaic device constructed at MIT [1] or the radiative cooling approach that was proposed at Stanford University [2,3].

In the same way, the realizations we present here deal with manipulating the spectral characteristics of nanostructures to achieve better energy efficiency in the context of home automation. We apply recent developments that we made in our research teams — at Institut des Nanotechnologies de Lyon (INL) and Institut d'Optique Graduate School (IOGS) - to the realization of incandescent infrared sources that have the ability to emit within controllable spectral range and solid angles.

Home automation represents in 2014 a vast market of ten billion of euros, with a growing rate of $10 \%$ per year. It deals with measures of temperature, humidity, smoke, or gas content in human habitation or industrial complexes. The measures are done by using sensors and ideally sensors that are wirelessly connected between each other. Ongoing costs include electricity to run the control systems, maintenance costs for the control and networking systems. In this context, there is a real need to lower the energy consumption of the sensors. In particular, gas sensors spend a high amount of energy owing to their constitutive incandescent IR source. In order to reduce the energy consumption, a filament exhibiting low thermal inertia must be chosen. The filament can be, that way, repeatedly heated within a few microseconds at each measure of the gas concentration. There exist mid infrared sources that exploit the high conductivity of platinum to reduce the power consumption well below 50 mW [4]. However, conventional incandescent sources are inherently inefficient owed to their broadband radiation, though the signature of molecules takes the form of narrow absorption rays. What is more, the thermal radiation of a bare object is omnidirectional. To improve the consumption, the power radiated in useless directions and wavelength ranges must be inhibited. Therefore, by only emitting within the spectral band of absorption of the molecule to be detected (e.g. around 4.26 microns for carbon dioxide) and in the direction of the detector, it is plain that the incandescent source would necessitate less energy to reach the required temperature.

In this communication, we employ photonic and plasmonic structures to achieve the coherent control of the emission of incandescent sources. We will present several resonant structures with engineered spectral properties in such a way that the emission of the device is that of the blackbody in the directional and spectral window of interest while the emissivity is remarkably low elsewhere. Our designs and experimental realizations are a response to energy efficiency concerns inasmuch as they are suitable candidates for the development, at the industrial level, of autonomous gas sensors, i.e., capable of lasting for several years with a simple AA commercial battery.

\section{Plasmonic approach for selective and directional thermal emission}

The control of the emission spectrum can be realized using a plasmonic approach based on subwavelength periodic array of metal-insulator-metal (MIM) cavities as drawn in Fig 1(a). It is well known that such structures consisting in a continuous back metallic mirror coated by a dielectric layer on which metallic square patches are structured, support a gap surface plasmon (GSP) mode [5]. Its resonance wavelength depends on the thickness of the dielectric layer and on the width of the top metallic square patch [6,7]. Thus the MIM cavities react as optical resonators able to absorb all incident light at the resonance, which corresponds to a peak of emissivity by means of Kirchoff's law. The physical mechanism of this key property can be understood as a critical coupling of incident plane waves to GSP resonators [8,9]. It is worth stressing that we demonstrate, this way, how to control the emission directivity thanks to diffraction [10].

More precisely, we design first a two-dimensional (2D) periodic array of MIM cavities in order to match the resonance at normal incidence with the $\mathrm{CO}_{2}$ absorption (near $2143 \mathrm{~cm}^{-1} \pm 60 \mathrm{~cm}^{-1}$ ). Then, we fix the period so that only the first order perturbation is diffracted from normal incidence to the required emission/incident angle.

Following this process, we have obtained the CMOS compatible metasurface configuration drawn in Fig 1(a). This structure has been realized in clean room and is represented in the SEM picture, Fig 1(b). The W backplane of thickness $200 \mathrm{~nm}$ is sputtered on a undoped Si wafer. Then, the SiN layer is deposited by Plasma Enhanced Chemical Vapor Deposition (PECVD). Finally, the top square patches are patterned by electron beam lithography with a lift-off process using the $100 \mathrm{~nm}$-thick Pt layer onto a $6 \mathrm{~nm}$-thick Ti adhesion layer. The emissivity measurement is performed by heating the sample at $500^{\circ} \mathrm{C}$ on a holder which is fixed on a motorized rotating goniometer. The emitted thermal radiation is collected with a 


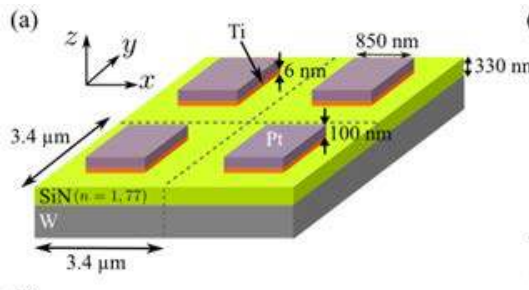

(b)

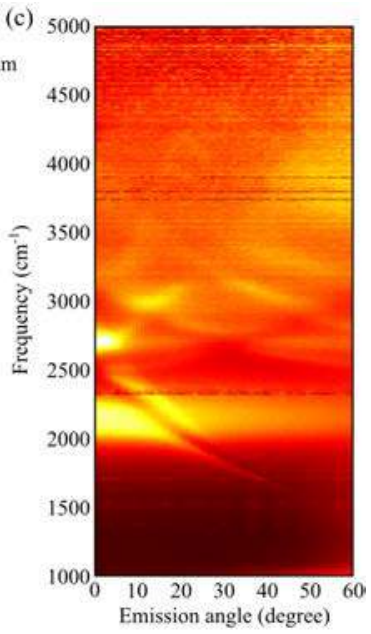

Fig. 1. (a) Layout of the plasmonic metasurface with the relevant geometrical parameters. (b) SEM picture of the realized structure. (c) Emission spectrum of the structure measured at $500^{\circ} \mathrm{C}$ and normalized by the blackbody spectrum. We obtain a $\left[0-15^{\circ}\right]$ spatial window in the spectral range of $\mathrm{CO}_{2}$ absorption $2143 \mathrm{~cm}^{-1} \pm 60 \mathrm{~cm}^{-1}$.

mirror system and sent into a FTIR spectrometer. By replacing the sample with a calibrated blackbody we could get an adequate reference in order to normalize our metasurface emissivity as shown in Fig 1(c).

We define the radiative efficiency as the ratio of the power emitted in the spectral and spatial window of interest to the total power emitted by the source. Our metasurface increases by a factor 2.4 the useful emission compared to the blackbody in the $\mathrm{CO}_{2}$ absorption spectral range and in the $\left[0-15^{\circ}\right]$ aperture range.

\section{Photonic approach for selective and directional thermal emission}

Photonic crystals (PCs) have come to constitute in the last decades a fundamental branch in the realm of nanophotonics. However the production at the industrial level of three-dimensional (3D) structures is inhibited by technological challenges (especially regarding the alignment of their constitutive elements). This is the reason why a particular attention has been dedicated to photonic crystal membranes (PCMs), also referred to as high contrast gratings (HCGs), which are dielectric wave guiding slabs with an in-plane texturization and an out-of-plane finite thickness [11]. Note in passing that their inherent vertical compactness makes them appealing in the context of integrated photonics.

The degree of confinement of photons inside PCMs may exhibit strong variation; therefore, PCMs can be used as broadband mirrors [12] while structures supporting extremely high quality $(Q)$ factor resonances were proposed and analyzed [13]. We have recently reported on a perturbation model that provides great insight into the mechanism presiding over the dramatic variation of the $Q$ factor [14,15]. According to this model the $Q$ factor is such that

$$
\begin{aligned}
\frac{1}{Q} & \propto \frac{\left[\rho_{21} \rho_{23}-1\right]\left[1+\rho_{21} \rho_{23}+\left(\rho_{21}+\rho_{23}\right) \cos d k_{2}\right]}{1+\rho_{21}^{2} \rho_{23}^{2}-2 \rho_{21} \rho_{23} \cos 2 d k_{2}} \times\left[\frac{\sin \frac{d}{2}\left(k_{2}-\kappa\right)}{k_{2}-\kappa}+\frac{\sin \frac{d}{2}\left(k_{2}+\kappa\right)}{k_{2}+\kappa}\right]^{2} \\
& =\frac{\sin \frac{d}{2}\left(k_{2}-\kappa\right)}{k_{2}-\kappa}+\frac{\sin \frac{d}{2}\left(k_{2}+\kappa\right)}{k_{2}+\kappa}=\left|\int_{0}^{d} \mathrm{e}^{i k_{2} x} \Phi(x) \mathrm{d} x\right|
\end{aligned}
$$

where $d$ is the membrane's thickness, $\rho_{21}$ and $\rho_{23}$ are the reflectivity at the interfaces, $k_{2}$ the wavenumber, $\kappa$ the vertical component of the guided mode propagation vector, and $\Phi(x)$ the vertical profile of the guided mode. It is fundamental to stress that all these quantities are related to the unperturbated membrane. A usual statement is to consider a resonance in a PCM as the result of the coupling between radiation and guided modes [16]. Through our perturbation model, we give an analytical justification to this perception by showing that the $Q$ factor is definitely proportional to the vertical overlap integral between the electromagnetic field distributions of these eigenmodes. Furthemore, it is plain from Eq. 1 that it is possible to get $Q \rightarrow \infty$ through a suitable choice of $d$.

On the one hand we have seen that the $Q$ factor can take very high values. As a result, provided that $d$ is such that $Q$ exhibits a high value, a slight perturbation of the former quantity results in a strong alteration of the latter. Since tilting the angle is equivalent to vary the optical thickness, the alteration of the $\mathrm{Q}$ factor can be achieved this way as well. On the other hand, the use of the critical coupling concept is the other ingredient towards selectivity and directionality by means of PCMs. In such a membrane we have a guided Bloch resonance that is addressable through the surface with a certain coupling rate. In addition to that, we may submit this resonance to losses via, for example, the presence of a metal or by chemical doping. The critical coupling conditions turn out to be realized when rates of coupling and losses are equal. Therefore, if we are in the critical coupling regime and if $Q$ is high enough, we understand that we can fastly get away from critical coupling through a variation of the incidence angle. According to Kirchhoff's law of thermal radiation the same phenomenon should occur if the device is heated.

In Fig. 2(a) is depicted a PCM, made up of etched silicium, that is separated from a gold layer by a certain spacer. The spacer control the degree of absorption, in the metal, of the confined photons. By adjusting PCM and spacer in such a way that (i) the structure resonates at $4.26 \mu \mathrm{m}$ and (ii) 


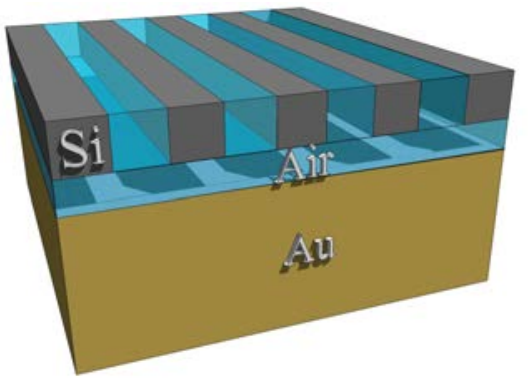

(a)

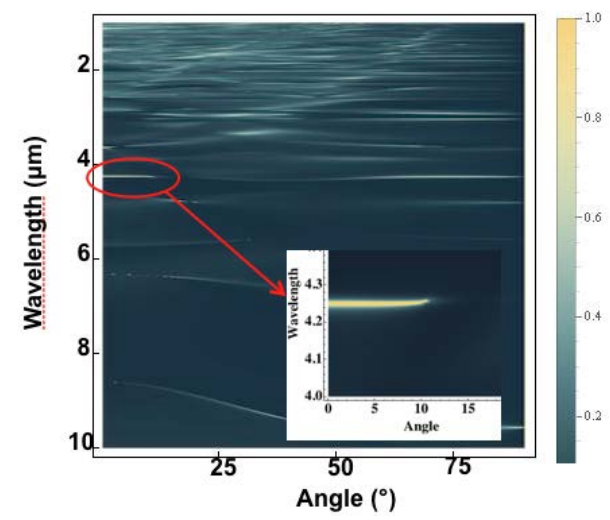

(b)

Fig. 2. (a) PCM (Si/Air) and metallic (Au) layer separated by an air gap. (b) Emissivity in terms of wavelength and angle. The emissivity is equal to unity at $4.26 \mu \mathrm{m}$ and from 0 to $10^{\circ}$.

the critical coupling condition regime is achieved, we obtain the emissivity numerically calculated, Fig. 2(b), in terms of wavelength and angle. In this configuration, compared to the blackbody at $600^{\circ} \mathrm{C}$, the total emission is 20 times lower. Nonetheless, only $15 \%$ of the black body radiation is emitted in the spectral and spatial windows of interest, viz. [4.16-4.36 $\mu \mathrm{m}]$ and $\left[0-15^{\circ}\right]$.

\section{Conclusion}

We have proposed two kinds of gas sensor with low energy consumption. The proposed structures are planar, are well-suited for integration and present no difficulty regarding the fabrication process. In the plasmonic approach we ensure the critical coupling to only happen for the fundamental order of diffraction while the other orders, which appear when the angle is increased, annihilate the critical coupling condition. In the photonic approach, the rates of coupling and losses are adjusted in such a way that the critical coupling regime is solely achieved around the spectral and spatial window of interest.

\section{Acknowledgments}

This work is part of the IDEE project which is funded by the French Agence Nationale de la Recherche (ANR). The authors acknowledge Laurent Carrel for helpful support regarding INL computing facilities.

\section{References}

1. J. A. Lenert et al., Nat. Nano., 9, 126-130, 2014.

2. E. Rephaeli et al., Nano Letters, 13(4), 1457-1461, 2013.

3. A. P. Raman et al., Nature, 515(7528), 540-544, 2014.

4. P. Barritault et al., Sens. Actuator A-Phys., 172(2), 379 - 385, 2011.

5. S. Collin et al., Opt. Express , 15, 4310, 2007.

6. P. Bouchon et al., Opt. Lett. , 37, 1038-1040, 2012.

7. H. T. Miyazaki et al., Appl. Phys. Lett., 105, 121107, 2014.

8. F. Pardo et al., Phys. Rev. Lett., 107, 93902, 2011.

9. B. J. Lee et al., Opt. Express, 16, 11328, 2008.

10. D. Costantini et al., submitted for publication, 2015.

11. S. G. Johnson et al., Phys. Rev. B, 60, 5751-5758, 1999.

12. H. Hattori et al., Opt. Express, 11(15), 1799-1808, 2003.

13. V. Karagodsky et al., Opt. Express, 20(10), 10888-10895, 2012.

14. C. Blanchard et al., Phys. Rev. A, 90(3), 033824, 2014.

15. C. Blanchard et al., in SPIE Photonics West 2015, 7-12 Feb. 2015.

16. R. Magnusson et al., Appl. Phys. Lett., 61(9), 1022-1024, 1992. 